\title{
ON THE COEFFICIENTS
}

\section{IN THE PRODUCT OF AN ALTERNANT}

\section{AND A SYMMETRIC FUNCTION*}

BI

E. D. ROE, JR.

\section{§1. Introduction. Notations.}

If with respect to $m$ indeterminates $\alpha_{1}, \ldots, \alpha_{m} A$ denote the simplest alternant $|01 \cdots m-1|$, viz. $\left|\alpha_{1}^{0} \alpha_{2}^{1} \cdots \alpha_{n}^{m-1}\right|, \phi$ a monomial symmetric function, and $A_{k}$ an alternant in their product

$$
A \phi=C_{1} A_{1}+C_{2} A_{2}+\cdots+C_{s} A_{s}, * *
$$

it was formerly necessary to find all of the $C$ 's in order to find one of them. The problem of obtaining a single $C$ alone was first considered by Murr $\dagger$ in a brief paper in 1899. His method was first to express $\phi$ by means of the symmetric function table in terms of products $p$ of weight $w$ of elementary symmetric functions, as

$$
\phi=c_{1} p_{1}+c_{2} p_{2}+\cdots+c_{r} p_{r} .
$$

This changes the problem to that of finding the $d$ 's in

$$
A p_{k}=d_{k, 1} A_{1}+d_{k, 2} A_{2}+\cdots+d_{k, s} A_{s} .
$$

If found, the $C$ 's are expressed by the relations

$$
C_{k}=c_{1} d_{1, k}+c_{2} d_{2, k}+\cdots+c_{r} d_{r, k} .
$$

MuIr obtained the expression for one particular $C, \ddagger$ in terms of the $c$ 's. Professor TAYLOR, proceeding from the standpoint of MuIr, has extended the solution, so as to obtain any $C$ in terms of the $c$ 's and $d$ 's. $\S$ Another method

* Presented to the Society December 29, 1902. Received for publication November 16, 1903.

** Cf. MurR, Theory of Determinants, p. 176, \$129.

$\dagger$ The Multiplication of an Alternant by a Symmetric Function of the Variables, Proceedings of the Royal Society of Edinburgh, 1898-99, pp. 539-542.

$\ddagger$ It is the relation (32) obtained in this paper.

§On the Product of an Alternant by a Symmetric Function, American Mathematical Monthly, vol. 10 (1903), pp. 119-130. 
of attacking this problem is that given by the writer.* It determines the $C^{\prime}$ 's directly from the original function without recourse to the elementary products and the symmetric function table. In fact it becomes simultaneously a method for expressing symmetric functions in terms of the elementary products. These two results were reached formally in a theorem

$$
|012 \cdots n-1| \sum \alpha_{1}^{p_{1}} \alpha_{2}^{p_{2}} \cdots \alpha_{m}^{p_{m}}=\sum\left\{\begin{array}{c}
p_{1} p_{2} \cdots p_{m} \\
i_{1} i_{2} \cdots i_{m}
\end{array}\right\}\left|i_{1} i_{2} \cdots i_{m}\right| \text {, }
$$

and in a related theorem,

$$
a_{0}^{n} \sum \alpha_{1}^{p_{1}} \alpha_{2}^{p_{2}} \cdots \alpha_{m}^{p_{m}}=(-1)^{w} \sum\left\{\begin{array}{l}
p_{1} p_{2} \cdots p_{m} \\
i_{1} i_{2} \cdots i_{m}
\end{array}\right\}\left\{i_{m+1} i_{m+2} \cdots i_{m+n}\right\} \cdot *
$$

(The notations are to be explained immediately.) The latter expresses a monomial symmetric function as a sum of determinants in the $a$ 's, the elementary symmetric functions. These results were obtained by using the resultant of two binary forms and the theory of corresponding matrices. $\dagger$

In this paper, which may be regarded as a continuation of my paper just cited, occur among other things: Direct proofs, without the use of the resultant, and for more general results when the alternant $\left|\lambda_{1} \lambda_{2} \cdots \lambda_{m}\right|$ is used as multiplier, $\S \S 2$ and 3 ; explicit methods for calculation and a generalized expression for the coefficients when the multiplier is $|012 \cdots m-1|, \S \S 3-6$; the $C$ 's in terms of the $c$ 's and the $d$ 's, which latter are also proved to be the coefficients in the quotient of two alternants, $\S 4$. It is proved that a table of $C^{\prime}$ 's for weight $w$ may be so arranged that it fills out a right triangle whose hypothenuse consists of ones, and the sum of whose every column except the last is zero, $\S 7$. The $d$ 's are expressed in terms of the $C$ 's, $\S 8$. The absolute invariance of the $C$ 's is proved when $|012 \cdots m+r-1|$ is the multiplier, $\S 9$. And it is shown, $\S 10$, how a two-fold application of the table gives any monomial symmetric function in terms of elementary products, so that this table is, in a certain sense, a square root of the usual symmetric function table.

A table of the $C^{\prime}$ 's for weight $w$ is called a product table for weight $w$. The theory involves certain general complexes of integers: $\lambda_{1} \lambda_{2} \cdots \lambda_{m}, \kappa_{1} \kappa_{2} \cdots \kappa_{m}$, $i_{1} i_{2} \cdots i_{m}, p_{1} p_{2} \cdots p_{m}$, etc.; these are denoted by $\lambda, \kappa, i, p$, etc., while the complex $\kappa_{i_{1}} \kappa_{i_{2}} \cdots \kappa_{i_{m}}$ is denoted by $\kappa_{i}$. The conjugate complexes $i_{1} i_{2} \cdots i_{m}$, and $i_{m+1} i_{m+2} \cdots i_{m+n}$ formed from each other, as previously explained, l. c.,* by taking those elements of the series $012 \cdots m+n-1$ which remain after the complementaries of the given elements with respect to $m+n-1$ have

* Note on Symmetric Functions, A merican Journal of Mathematics, vol. 25 (1903), pp. 97-106.

† GORDAN, Invariantentheorie, vol. 1, p. 95. 
been rejected, are denoted by $i$ and $\bar{\imath}$. We have $\overline{\bar{\imath}}=i$. If $n=m, i$ and $\bar{\imath}$ are principal conjugates according to $\S 7,1$.

The functions and the coefficients to be studied have the following definitions and notations :

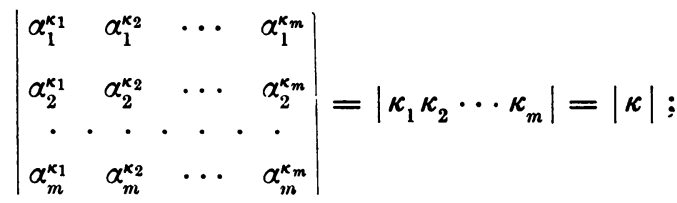

$$
\begin{aligned}
& \left|\kappa_{i_{1}} \kappa_{i_{2}} \cdots \kappa_{i_{m}}\right|=\left|\kappa_{i}\right| .
\end{aligned}
$$

(8)

$$
\left|\begin{array}{cccc}
a_{\kappa_{1}-\lambda_{1}} & a_{\kappa_{2}-\lambda_{1}} & \cdots & a_{\kappa_{m}-\lambda_{1}} \\
a_{\kappa_{1}-\lambda_{2}} & a_{\kappa_{2}-\lambda_{2}} & \cdots & a_{\kappa_{m}-\lambda_{2}} \\
\cdot & \cdot & \cdot & \cdot \\
a_{\kappa_{1}-\lambda_{m}} & a_{\kappa_{2}-\lambda_{m}} & \cdots & a_{\kappa_{m}-\lambda_{m}}
\end{array}\right|=\left\{\kappa_{1}-\lambda_{1} \kappa_{2}-\lambda_{1} \cdots \kappa_{m}-\lambda_{1}\right\}=\{\kappa-\lambda\}
$$

$$
\begin{gathered}
=\left[\kappa_{1}-\lambda_{1} \kappa_{2}-\lambda_{2} \cdots \kappa_{m}-\lambda_{m}\right] . \\
\sum \alpha_{1}^{p_{1}} \alpha_{2}^{p_{2}} \cdots \alpha_{m}^{p_{m}}=\left(p_{1} p_{2} \cdots p_{m}\right)=(p) \\
\left(\sum \alpha_{1} \alpha_{2} \cdots \alpha_{j_{1}}\right)^{i_{1}}=\left(1^{j_{1}}\right)^{i_{1}} \\
\left(1^{j_{1}}\right)^{i_{1}}\left(1^{j_{2}}\right)^{i_{2}} \cdots\left(1^{j_{m}}\right)^{i_{m}}=\left(1^{j}\right)^{i} .
\end{gathered}
$$

$$
\left|\begin{array}{c}
\kappa_{1} \kappa_{2} \cdots \kappa_{m} \\
\lambda_{1} \lambda_{2} \cdots \lambda_{m} p_{1} p_{2} \cdots p_{m}
\end{array}\right|=\left|\begin{array}{c}
\kappa \\
\lambda p
\end{array}\right|=\text { the coefficient of }|\kappa| \text { in }|\lambda|(p) .
$$

$$
\left|\begin{array}{c}
\kappa_{1} \kappa_{2} \cdots \kappa_{m} \\
\lambda\left(1^{j_{1}}\right)^{i_{1}}\left(1^{j_{2}}\right)^{i_{2}} \cdots\left(1^{j_{m}}\right)^{i_{m}}
\end{array}\right|=\left|\begin{array}{c}
\kappa \\
\lambda\left(1^{j}\right)^{i}
\end{array}\right|=\text { the coefficient of }|\kappa| \text { in }|\lambda|\left(1^{j}\right)^{i} .
$$

$$
\left\|\begin{array}{c}
p_{1} p_{2} \cdots p_{m} \\
\kappa_{1} \kappa_{2} \cdots \kappa_{m} / \lambda_{1} \lambda_{2} \cdots \lambda_{m}
\end{array}\right\|=\left\|\begin{array}{c}
p \\
\kappa / \lambda
\end{array}\right\|=\text { the coefficient of }(p) \text { in }|\kappa| /|\lambda| \text {. }
$$

$$
\begin{gathered}
\left\{\begin{array}{c}
p_{1} p_{2} \cdots p_{m} \\
\kappa_{1}-\lambda_{1} \kappa_{2}-\lambda_{1} \cdots \kappa_{m}-\lambda_{1}
\end{array}\right\}=\left[\begin{array}{c}
p_{1} p_{2} \cdots p_{m} \\
\kappa_{1}-\lambda_{1} \kappa_{2}-\lambda_{2} \cdots \kappa_{m}-\lambda_{m}
\end{array}\right] \\
=\left\{\begin{array}{c}
p \\
\kappa-\lambda
\end{array}\right\}=\text { the coefficient of } a_{p_{1}} a_{p_{2}} \cdots a_{p_{m}}=a_{p} \text { in }\{\kappa-\lambda\} .
\end{gathered}
$$

$$
\left(\begin{array}{c}
p_{1} p_{2} \cdots p_{m} \\
0^{m} \kappa_{1} \kappa_{2} \cdots \kappa_{m}
\end{array}\right)=\left(\begin{array}{c}
p \\
\kappa
\end{array}\right)=\text { the coefficient of } a_{p_{1}} a_{p_{2}} \cdots a_{p_{m}}=a_{p} \text { in } a_{0}^{m}(\kappa) .
$$

The notations in (10), (11) and (12) will usually be written in this paper with the complex $\lambda$ omitted, the known and definite $\lambda$ so omitted being in each case specified in the context. Thus (10) and (12) are written $\left|\begin{array}{l}\kappa_{1} \kappa_{2} \ldots \kappa_{m} \\ p_{1} p_{2} \ldots p_{m}\end{array}\right|=\left|\begin{array}{l}\kappa \\ p\end{array}\right|$,

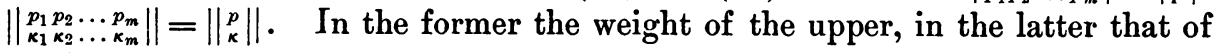
the lower indices, is greater. 


\section{§ 2. The Theorem $\left|\begin{array}{c}{ }^{\alpha} p \\ \lambda_{p}\end{array}\right|=\left\{\begin{array}{c}p \\ \kappa-\lambda\end{array}\right\}$.}

We shall prove that $\left|\begin{array}{c}{ }^{k} \\ \lambda^{\prime}\end{array}\right|=\left\{\begin{array}{c}p \\ \kappa-\lambda\end{array}\right\}$. This is seen by considering the mode of formation of $\left|{ }_{\lambda p}{ }^{\alpha}\right|$. In order to obtain it we add a permutation $p_{i}$ of the $p$ 's to $\lambda$ and get,

$$
p_{i_{1}}+\lambda_{1}, p_{i_{2}}+\lambda_{2}, \cdots, p_{i_{m}}+\lambda_{m} .
$$

If these numbers are all different and a permutation of $\kappa$, we have one of the alternants sought, namely

$$
\left|\kappa_{i}\right|=(-1)^{i}|\kappa|
$$

and where the exponent $i$ is the number of inversions of order in $\kappa_{i}=\kappa_{i_{1}} \kappa_{i_{2}} \cdots \kappa_{i_{m}}$ from the order $\kappa=\kappa_{1} \kappa_{2} \cdots \kappa_{m}$ of ascending magnitude, i. e., $i$ is the number of inversions of order among $i_{1}, i_{2}, \cdots i_{m}$. Then it is evident that

$$
\left|\begin{array}{c}
\kappa \\
\lambda p
\end{array}\right|=\sum(-1)^{i} \text {. }
$$

The number of terms in $\sum$ is the number of complete sets of solutions of the equations (16). To define this number we write

$$
\begin{aligned}
& \kappa_{1}-\lambda_{1} \quad \kappa_{2}-\lambda_{1} \quad \cdots \quad \kappa_{m}-\lambda_{1} \\
& \kappa_{1}-\lambda_{2} \quad \kappa_{2}-\lambda_{2} \quad \cdots \quad \kappa_{m}-\lambda_{2} \\
& \kappa_{1}-\lambda_{m} \quad \kappa_{2}-\lambda_{m} \quad \cdots \quad \kappa_{m}-\lambda_{m}
\end{aligned}
$$

and observe that according to (1.6) each $\kappa$ in the complex $\kappa_{i}$ comes from the addition of a $\kappa-\lambda_{i}$ in the $i$ th line of the array (18), to the $i$ th number in the complex $\lambda$, provided that $\kappa-\lambda_{i}$ is one of the $p$ 's. Then to form a permutation of the $\kappa$ 's, only one number can be added from the $i$ th line to $\lambda_{i}$, and also only one number can be taken from the $i$ th column. Hence a permutation of $\kappa_{1} \kappa_{2} \cdots \kappa_{m}$ can be formed in as many ways as we can select one and only one number from each line and column of the array (18), with the restriction that the numbers selected form a permutation of the $p$ 's. Let the element selected from the $r$ th line be $\kappa_{i_{r}}-\lambda_{r}$. Then the selected elements correspond to the substitution $\left(\begin{array}{l}1 \\ i_{1} i_{2} i_{3} \ldots i_{m}\end{array}\right)$ with modulus $(-1)^{i}$. If we select a term $a_{p}$ from the determinant $\{\kappa-\lambda\}$, one such term would, from the foregoing, evidently be obtained by selecting the elements from the determinant according to the substitution $\left(\begin{array}{l}1 \\ i_{1} i_{2} \\ i_{2} i_{3} \ldots i_{m}\end{array}\right)$ and the sign of this term would also be $(-1)^{i}$. Hence to every alternant $\left|\kappa_{i}\right|=(-1)^{i}|\kappa|$, corresponds a term $(-1)^{i} a_{p}$ of the determinant $\{\kappa-\lambda\}$, and conversely, and hence 
and therefore by (17)

$$
\left\{\begin{array}{c}
p \\
\kappa-\lambda
\end{array}\right\}=\sum(-1)^{i}
$$

$$
\left|\begin{array}{c}
\kappa \\
\lambda p
\end{array}\right|=\left\{\begin{array}{c}
p \\
\kappa-\lambda
\end{array}\right\}
$$

If $\lambda=012 \cdots m-1$, this relation becomes

$$
\left|\begin{array}{c}
\kappa \\
p
\end{array}\right|=\left\{\begin{array}{c}
p \\
\kappa
\end{array}\right\}
$$

\section{§3. On the calculation of $\left|{ }_{\lambda p}^{\kappa}\right|$.}

1. In the paper previously cited (p. 2) a reduction formula was given for coefficients when the multiplier is $|012 \cdots m-1|$. It is easy to see what this formula becomes for the general case. Put $\kappa_{r}-\lambda_{1}=\sigma_{r}$. If $r$ numbers be common to the two complexes $p, \sigma$, so that

$$
\sigma_{\mu_{1}}=p_{\nu_{1}}, \sigma_{\mu_{2}}=p_{\nu_{2}}, \cdots, \sigma_{\mu_{r}}=p_{\nu_{r}},
$$

we have by expanding $\{\sigma\}$ in terms of the elements of its first line

(22) $\left\{\begin{array}{c}p \\ \sigma\end{array}\right\}=\sum_{\kappa=1}^{\kappa=r}(-1)^{\mu_{\kappa}-1}\left\{\begin{array}{llll}p_{\nu_{1}} & p_{\nu_{2}} \cdots p_{\nu_{\kappa-1}} & p_{\nu_{\kappa+1}} & \cdots p_{\nu_{m}} \\ \sigma_{1}-\tau & \sigma_{2}-\tau \cdots \sigma_{\mu_{\kappa-1}}-\tau & \sigma_{\mu_{\kappa+1}}-\tau \cdots \sigma_{m}-\tau\end{array}\right\}$, where $\tau=\lambda_{2}-\lambda_{1}$. In general in the $r$ th stage of reduction we subtract $\lambda_{r+1}-\lambda_{r}$ from all the elements remaining in the lower line. If no numbers of the two series are common, or if a lower index becomes negative, the coefficient is zero. For example, to find $\left|{ }_{0213}^{2459}\right|$ with $\lambda_{1}=2, \lambda_{2}=3, \lambda_{3}=5, \lambda_{4}=6$, we have,

$$
\left|\begin{array}{l}
2459 \\
0^{2} 13
\end{array}\right|=\left\{\begin{array}{l}
0^{2} 13 \\
0237
\end{array}\right\}=\left\{\begin{array}{l}
013 \\
126
\end{array}\right\}=\left\{\begin{array}{l}
03 \\
04
\end{array}\right\}=\left\{\begin{array}{l}
3 \\
3
\end{array}\right\}=1 \text {. }
$$

2. The calculation is shortened by changing the notation slightly. Instead of the first line, the principal diagonal indices $\kappa_{1}, \kappa_{2}-1, \cdots, \kappa_{m}-m+1$, (for $\lambda=012 \cdots m-1)$ may be used. They are the different partitions of weight $m$. Thus for weight 5 we have the partitions:

00005

00014

00023

00122

01112

Taking each one with all the others in order from below upwards, one obtains all the coefficients of the table for weight 5 . Thus the 11111 


$$
\left[\begin{array}{l}
00005 \\
11111
\end{array}\right]=\left\{\begin{array}{l}
00005 \\
12345
\end{array}\right\}=\left|\begin{array}{l}
12345 \\
00005
\end{array}\right|
$$

where $[11111]=\{12345\}$.

The value of $\left[\begin{array}{c}p_{1} p_{2} \ldots p_{m} \\ i_{1} i_{2} \ldots i_{m}\end{array}\right]$ is the number of ways in which the lower complex, $i$, can be made equal to the upper, $p$, by the following operation: passing a lower number from right to left over an adjacent number introduces the factor $(-1)$ towards the result, reduces the number passed over by one, and increases that passing over by one. The coefficient is the algebraic sum of the number of distinct ways in which the complex $i$ can thus be made equal to the complex $p$. E. g.

$\left[\begin{array}{l}00005 \\ 11111\end{array}\right]=(-1)^{4}$. The right hand unit, passed over the preceding four, produces four zeros and a five; and this result can be produced in no other way by the process described.

$\left[\begin{array}{l}00113 \\ 1111\end{array}\right]=3$. Here, as two zeros and a three must be obtained, unity must be passed over two units. This can be done in three ways, by passing the $3 \mathrm{~d}$, 4 th or th th unit over two preceding ones.

$\left[\begin{array}{l}00122 \\ 1111\end{array}\right]=3$. (2d and 4 th) or (3d and 5 th) or ( $2 \mathrm{~d}$ and 5 th) over one preceding unit.

$\left[\begin{array}{l}00014 \\ 1111\end{array}\right]=-2 . \quad 4$ th or 5 th over three preceding units.

The theory of this is simply that the operations performed represent the different ways in which the term in question can be brought to the principal diagonal of the determinant involved, and it becomes clear by considering the effect on the indices when one column is passed over another at its left.

If $|\lambda|$ is used as multiplier, the factor $(-1)$ is introduced as before, but if the element in the $r$ th place pass over that in the $(r-1)$ th, that passing over is increased, while that passed over is decreased by $\lambda_{r}-\lambda_{r-1}$. For example with $\lambda_{1}=2, \lambda_{2}=3, \lambda_{3}=5, \lambda_{4}=6,\left|\begin{array}{l}2567 \\ 0013\end{array}\right|=\left\{\begin{array}{l}0013 \\ 0345\end{array}\right\}=\left[\begin{array}{l}0013 \\ 0211\end{array}\right]=-1$, for in moving 1 in the third place over 2 , the former must be increased and the latter diminished by 2 , and this is the only way of making the two complexes alike. It is to be especially noted that the difference $\lambda_{r}-\lambda_{r-1}$ belongs to the rth place whatever the elements moved.

\section{$\S 4$ Relations between the Coefficients $\left.\right|_{p}{ }_{p} \mid$ and the Coefficients in the Symmetric Function Table.}

In the paper already cited (p. 2) the relation

or,

$$
\left(\begin{array}{l}
q_{1} q_{2} \cdots q_{n} \\
0^{n} p_{1} p_{2} \cdots p_{m}
\end{array}\right)=(-1)^{w} \sum_{i}\left|\begin{array}{l}
i_{1} i_{2} \cdots i_{m} \\
p_{1} p_{2} \cdots p_{m}
\end{array}\right|\left|\begin{array}{l}
i_{m+1} \cdots i_{m+n} \\
q_{1} q_{2} \cdots q_{n}
\end{array}\right|
$$

$$
\left(\begin{array}{l}
q \\
p
\end{array}\right)=(-1)^{w} \sum_{i}: \begin{array}{ll}
i \\
p
\end{array}\left|\begin{array}{l}
\bar{\imath} \\
q
\end{array}\right|,
$$


where $p$ and $q$ are of weight $w, i$ of weight $w+\frac{1}{2} m(m-1)$, and $\bar{\imath}$ of weight $w+\frac{1}{2} n(n-1)$, was given.

We shall now express $\left|\begin{array}{l}i \\ p\end{array}\right|$ in terms of the $\left(\begin{array}{c}p \\ k\end{array}\right)$ and the $\left\|{ }_{i}^{\kappa}\right\|$, where the complexes $p$ and $\kappa$ are of weight $w, i$ is of weight $w+\frac{1}{2} m(m-1)$, and $\bar{\imath}$ is of weight $w+\frac{1}{2} n(n-1)$.

By using the theorem of corresponding matrices and by the use of the matrices employed in the former paper and the additional matrix

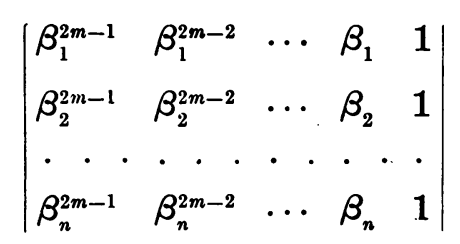

where the $\beta$ 's are the roots of

$$
\phi(x)=b_{0} x^{n}+b_{1} x^{n-1}+\cdots+b_{n}=0,
$$

we have, for $\lambda=012 \cdots m-1$,

$$
\{i\}=(-1)^{\mu} \sigma\left|i_{m+n} i_{m+n-1} \cdots i_{m+1}\right|,
$$

where

$$
\begin{aligned}
\mu=\frac{m(m+1)}{2}+i_{1}+1+i_{2}+1+\cdots+i_{m}+1=m(m+1) & +w, \\
& \equiv w(\bmod 2) .
\end{aligned}
$$

Also

therefore

$$
\sigma=\frac{b_{0}^{m}}{|n-1, n-2, \cdots, 1,0|},
$$

$$
\{i\}=(-1)^{w} b_{0}^{m} \frac{\left|i_{m+1} i_{m+2} \cdots i_{m+n}\right|}{|0,1, \cdots, n-1|},
$$

and hence

$$
\left\{\begin{array}{c}
p \\
i
\end{array}\right\}=\text { the coefficient of } b_{p} \text { in }(-1)^{w} b_{0}^{m} \frac{\left|i_{m+1} \cdots i_{m+n}\right|}{|0,1, \cdots, n-1|} .
$$

Since

$$
i_{m+1}+\cdots+i_{m+n}=\frac{n(n-1)}{2}+w,
$$

$\left|i_{m+1} \cdots i_{m+n}\right| /|0,1, \cdots, n-1|$ is a linear function of monomial symmetric functions of weight $w$, and from the quotient of this division one infers directly the expression for $\left|{ }_{p}^{i}\right|$ as a linear function of the coefficients in the different symmetric functions of weight $w$ (cf. $\S 8$ ). Hence 


$$
\frac{\left|i_{m+1} \cdots i_{m+n}\right| \cdot}{|0,1, \cdots, n-1|}=\sum_{\kappa} \| \begin{gathered}
\kappa_{1} \kappa_{2} \cdots \kappa_{n} \\
i_{m+1} \cdots i_{m+n}
\end{gathered} \mid\left(\kappa_{1} \kappa_{2} \cdots \kappa_{n}\right),
$$

and since $\left(\begin{array}{l}p \\ \kappa\end{array}\right)=\left(\begin{array}{l}k \\ p\end{array}\right)$, we have

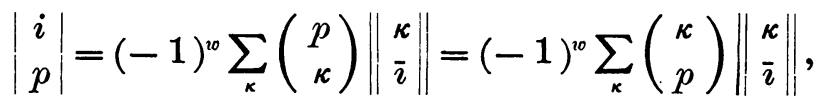

where the complex $\kappa$ begins with $i_{m+1}, i_{m+2}-1, \cdots i_{m+n}-n+1$, and then runs through each succeeding partition (containing $n$ elements) $\dagger$ in the sense of $\S 7,2$. A table containing coefficients $\left\|{ }_{i}\right\|$ for weight $w$, is called a quotient table for weight $w . \ddagger$ What has just preceded shows that Dr. TAYLOR's product table is the author's quotient table, with conjugate columns interchanged, $\S$ for it gives the multiplier $\left\|{ }_{\imath}\right\|$ by which the symmetric function $(\kappa)$ and therefore the individual coefficients in its expression in terms of elementary symmetric functions are to be multiplied in order to obtain $\left|\begin{array}{l}i \\ p\end{array}\right|$. But this is the coefficient which Dr. TAYLOR seeks. The exact relation between the two, using the writer's notation, may therefore be expressed as follows :

$$
\left\|\begin{array}{c}
1^{x_{1}} 2^{x_{2}} \cdots n^{x_{n}} \\
i_{m+1} \cdots i_{m+n}
\end{array}\right\|=\left|\begin{array}{c}
i_{1} i_{2} \cdots i_{m} \\
(1)^{x_{1}}\left(1^{2}\right)^{x_{2}} \cdots\left(1^{n}\right)^{x_{n}}
\end{array}\right|,
$$

where the coefficient on the left is a coefficient in the author's quotient table, that on the right, one in Dr. TAYLOR's product table. We have in fact

by (4) and

$$
\left|\begin{array}{c}
i \\
p
\end{array}\right|=(-1)^{w 0} \sum_{\kappa}\left(\begin{array}{c}
\kappa \\
p
\end{array}\right)\left|\begin{array}{c}
i \\
\left(1^{\kappa}\right)
\end{array}\right| \text {, }
$$

$$
\left|\begin{array}{l}
i \\
p
\end{array}\right|=(-1)^{i c} \sum_{\kappa}\left(\begin{array}{l}
\kappa \\
p
\end{array}\right)\left\|\begin{array}{c}
\kappa \\
i
\end{array}\right\|,
$$

by (29). Putting the two expressions for $\left|{ }_{p}^{i}\right|$ equal to each other, and equating coefficients of $\left(\begin{array}{l}\kappa \\ p\end{array}\right)$ and writing $\kappa$ in the form $1^{x_{1}} 2^{x_{2}} \cdots n^{x_{n}}$ we have the relation (30), as stated. By using a theorem proved in $\S 7,3$, that the sum of the coefficients of every column in the table for weight $w$ is zero, except for the last column, which contains only unity, it follows that in particular

$$
\frac{|0,1,2, \cdots, m-2,2 m-1|}{|0,1,2, \cdots, m-1|}
$$

* This is the CAyley-Betri law of symmetry. See CAyley, Collected Mathematical Papers, vol. 2, p. 421. The writer has given a proof of it in the American Mathematical Monthly, vol. 6 (1899), p. 104.

tThe number $n$ is the order of $(p)$.

$\ddagger$ The author reserves for a future paper a consideration of the properties of such coefficients similar to that of the present paper for the coefficients of the product table.

\& Since the complexes $i=i_{1} i_{2} \cdots i_{m}$ and $\bar{\imath}=i_{m+1} \cdots i_{m+n}$ of $(26)$ are conjugate to each other in the sense of $\S 7,1$. 
equals the sum of all monomial symmetric functions of weight $w$. Hence for any coefficient in the first column we have,

$$
\left|\begin{array}{c}
123 \cdots m \\
p_{1} p_{2} \cdots p_{m}
\end{array}\right|=(-1)^{w} \sum_{\kappa}\left(\begin{array}{l}
p \\
\kappa
\end{array}\right) \quad\left(\kappa_{1}+\kappa_{2}+\cdots+\kappa_{m}=w\right)
$$

or, by the Cayley-Betti law,

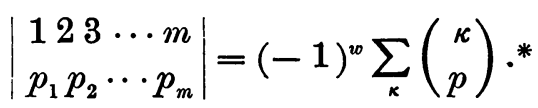

The reader may readily verify the foregoing discussion by reference to a table of symmetric functions in connection with the tables here given. Thus he will find :

$\left|\begin{array}{c}1234567 \\ 0^{5} 16\end{array}\right|=-(7-1-7-7+1+8+4-1+7-9+1-5+5-1)=-2$.

In a similar manner we have by performing the divisions,

$$
\begin{aligned}
\frac{|0123510|}{|012345|}=(51)+(42)+\left(3^{2}\right)+ & 2\left(41^{2}\right)+2(321)+3\left(31^{3}\right) \\
& +2\left(2^{3}\right)+3\left(2^{2} 1^{2}\right)+4\left(21^{4}\right)+5\left(1^{6}\right), \\
\frac{|012369|}{|012345|}=(42)+\left(3^{2}\right)+\left(41^{2}\right)+2(321)+ & 3\left(31^{3}\right)+3\left(2^{3}\right) \\
& +4\left(2^{2} 1^{2}\right)+6\left(21^{4}\right)+9\left(1^{6}\right),
\end{aligned}
$$

and these give the multipliers for forming the coefficients, in the product, by the symmetric function table, in the second and third columns of our table for weight six. Thus : $\left|{ }_{0415}^{023457}\right|=$ coefficient of $b_{0}^{4} b_{1} b_{5}$ in

$$
\begin{gathered}
b_{0}^{6} \frac{|0123510|}{|012345|}=-1-6-3+2.1+2.7+3(-1)+2.2+3(-4)+4.1=-1, \\
\left|\begin{array}{c}
013467 \\
0^{4} 24
\end{array}\right|=2-3+2+2.4+3(-2)+3(-2)+4.1=1 .
\end{gathered}
$$

By $\S 3,2$, for confirmation and comparison of methods,

$$
\left|\begin{array}{c}
023457 \\
0^{4} 15
\end{array}\right|=\left\{\begin{array}{c}
0^{4} 15 \\
023457
\end{array}\right\}=\left[\begin{array}{l}
000015 \\
011112
\end{array}\right]=-1,
$$

the latter result, since 2 must pass over three elements to the left, and this is the only way of making both complexes equal. $\left|\begin{array}{c}013467 \\ 0424\end{array}\right|=\left\{\begin{array}{c}0424 \\ 013467\end{array}\right\}=\left[\begin{array}{c}000024 \\ 001122\end{array}\right]=1$,

* This is the relation obtained by MurR. Its explicit expression is obtained in this paper in $\S 6,(44)$. See also $\S 6,(45)$. 
since 2 in the lower complex passes over the two preceding units, to make the two complexes equal.

\section{\$5. Relations between the t's and the a's.}

By $t_{w}$ we denote the sum of all the monomial symmetric functions of weight $w$. Then one has

$$
\begin{gathered}
\sum_{0}^{n} a_{r} x^{r}=a_{0} \prod_{1}^{n}\left(1-a_{r} x\right), \\
\frac{1}{\sum_{0}^{n} a_{r} x^{r}}=\frac{1}{a_{0}} \sum_{0}^{\infty} t_{r} x^{r},
\end{gathered}
$$

as identities in the $a$ 's, the $x^{\prime}$ s and $x$. From (34), cleared of fractions, by equating the coefficients of $x^{n}$,

$$
a_{0} t_{n}+a_{1} t_{n-1}+\cdots+a_{n} t_{0}=0, *
$$

whence

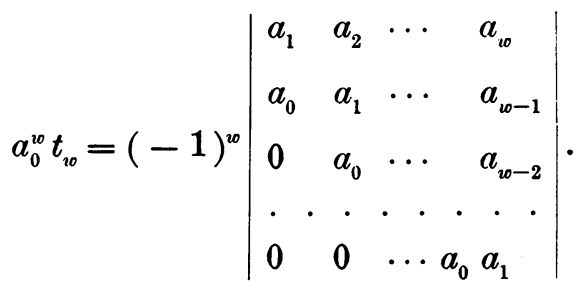

By developing the left member of (34) again in terms of $a$ 's and $x$ and equating coefficients of $x^{w}$, we have

$$
a_{0}^{w 0} t_{w}=\sum(-1)^{\lambda_{1}+\lambda_{2}+\cdots+\lambda_{w}} \frac{\left(\lambda_{1}+\lambda_{2}+\cdots+\lambda_{w}\right) !}{\lambda_{1} ! \lambda_{2} ! \cdots \lambda_{w} !} a_{0}^{\lambda_{0}} a_{1}^{\lambda_{1}} \cdots a_{w}^{\lambda_{w}}, \dagger
$$

where $\lambda_{0}+\lambda_{1}+\cdots+\lambda_{w}=\lambda_{1}+2 \lambda_{2}+\cdots+w \lambda_{w}=w$. From (36) and (37), we get,

$$
\{12 \cdots w\}=(-1)^{w} \sum(-1)^{\lambda_{1}+\lambda_{2}+\cdots+\lambda_{w}} \frac{\left(\lambda_{1}+\lambda_{2}+\cdots+\lambda_{w}\right) !}{\lambda_{1} ! \lambda_{2} ! \cdots \lambda_{w} !} a_{0}^{\lambda_{0}} a_{1}^{\lambda_{1}} \cdots a_{w}^{\lambda_{w}} .
$$

In MurR's Determinants, $\ddagger$ it is shown that

* Cf. Burnside and Panton, Theory of Equations, second edition, p. 297.

$\dagger$ By the symmetry of (35), $a_{w}$ is the same function of the $t^{\prime} \mathrm{s}$, that $t_{w}$ is of the $a^{\prime} \mathrm{s}$, and we note two expressions for the $a$ 's similar to (36) and (37).

$\ddagger$ Edition of 1882, p. 170, §125. 
(39)

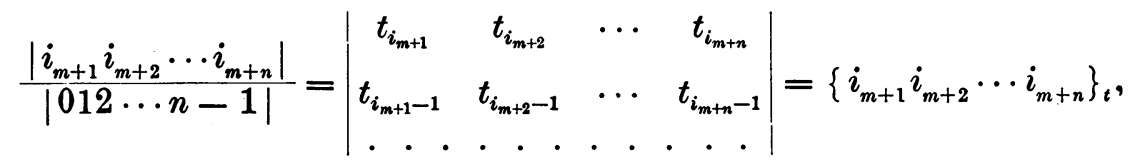

where the meaning of the subscript $t$ is obvious. Hence

or

$$
\left|\begin{array}{c}
i_{1} i_{2} \cdots i_{m} \\
p_{1} p_{2} \cdots p_{m}
\end{array}\right|=(-1)^{w} a_{0}^{m}\left\{\begin{array}{l}
p_{1} p_{2} \cdots p_{m} \\
i_{m+1} \cdots i_{m+n}
\end{array}\right\}_{t}
$$

$$
\left|\begin{array}{l}
i \\
p
\end{array}\right|=(-1)^{w 0} a_{0}^{m}\left\{\begin{array}{l}
p \\
\bar{\imath}
\end{array}\right\}_{t} \text {. }
$$

We have, as an important inference from the preceding relations,

whence

$$
a_{0}^{m}\left\{i_{m+1} \cdots i_{m+n}\right\}_{t}=(-1)^{w}\left\{\imath_{1} i_{2} \cdots i_{m}\right\}_{a},
$$

$$
\begin{aligned}
& a_{0}^{m}\{i\}_{t}=(-1)^{w 0}\{\bar{i}\}_{a}, \\
& a_{0}^{m}\{\bar{i}\}_{t}=(-1)^{w}\{i\}_{a} .
\end{aligned}
$$

This leads us to inquire whether, more generally,

$$
a_{0}^{m}\left\{i_{m+1}-\lambda_{1} \cdots i_{m+n}-\lambda_{1}\right\}_{t}=(-1)^{w}\left\{i_{1}-\lambda_{1} \cdots i_{m}-\lambda_{1}\right\}_{a} .
$$

By multiplying the right hand determinant by $\mu^{\Sigma_{1}^{m}\left(i_{r}-\lambda_{r}\right)}$, (the $r$ th column by $\mu^{i_{r}-\lambda_{1}}$; the $r$ th line, except the first, by $\mu^{\lambda_{1}-\lambda_{r}}$ ), we have, denoting the determinant by $f$,

$$
\mu_{\mu^{1}}^{\sum_{(i-\lambda)}} f\left(a_{0}, a_{1}, \cdots a_{m}\right)=f\left(\mu^{0} a_{0}, \mu^{1} a_{1}, \ldots \mu^{m} a_{m}\right) ;
$$

hence $f$ is isobaric and of weight

$$
\sum_{r=1}^{r=m}\left(i_{r}-\lambda_{r}\right)
$$

Similarly the left hand determinant is isobaric and of weight

$$
\sum_{r=m+1}^{r=m+n} i_{r}-\sum_{r=1}^{r=n} \lambda_{r} .
$$

That equality may exist, it is at least necessary that

$$
\sum_{r=1}^{r=m}\left(i_{r}-\lambda_{r}\right)=\sum_{r=m+1}^{r=m+n} i_{r}-\sum_{r=1}^{r=n} \lambda_{r} .
$$

Since, as previously proved (l. c., p. 2), 
this requires

$$
\sum_{r=1}^{r=m} i_{r}-\frac{m(m-1)}{2}=\sum_{r=m+1}^{r=m+n} i_{r}-\frac{n(n-1)}{2},
$$

$$
(m+n-1)(m-n)=2 \sum_{n+1}^{m} \lambda_{r}
$$

which is not generally true, unless $n=m$, when it subsists, as $\lambda_{m+1}=\lambda_{m+2}=\cdots=0$. But that the proposed relation is not generally true even when $n=m$, is seen by taking $m=n=4, \lambda_{1}=2, \lambda_{2}=3, \lambda_{3}=5, \lambda_{4}=6$. Then, $(i=2,5,6,7)$,

$$
a_{0}^{4}\left|\begin{array}{cccc}
t_{1} & t_{2} & t_{4} & t_{5} \\
t_{0} & t_{1} & t_{3} & t_{4} \\
0 & 0 & t_{1} & t_{2} \\
0 & 0 & t_{0} & t_{1}
\end{array}\right|=a_{0}^{4}\left|\begin{array}{cc}
t_{1} & t_{2} \\
t_{0} & t_{1}
\end{array}\right|, \quad\left|\begin{array}{cccc}
a_{0} & a_{3} & a_{4} & a_{5} \\
0 & a_{2} & a_{3} & a_{4} \\
0 & a_{0} & a_{1} & a_{2} \\
0 & 0 & a_{0} & a_{1}
\end{array}\right|=a_{0}\left|\begin{array}{ccc}
a_{2} & a_{3} & a_{4} \\
a_{0} & a_{1} & a_{2} \\
0 & a_{0} & a_{1}
\end{array}\right| .
$$

Therefore equality is impossible as the latter determinant contains $a_{\downarrow}$, while the former does not.*

\section{§6. A general expression for $\left|\begin{array}{l}i \\ p\end{array}\right|$.}

Using (38) and (41) and putting the complex $p_{1} p_{2} \cdots p_{m}=0^{x_{0}} 1^{x_{1}} 2^{x_{2}} \cdots m^{\dot{x}_{m}}$, we have

$$
\left|\begin{array}{c}
12 \cdots m \\
p_{1} p_{2} \cdots p_{m}
\end{array}\right|=\left\{\begin{array}{c}
0^{x_{0}} 1^{x_{1}} \cdots m^{x_{m}} \\
12 \cdots m
\end{array}\right\}=(-1)^{m+x_{1}+x_{2}+\cdots+x_{m}} \frac{\left(x_{1}+x_{2}+\cdots+x_{m}\right) !}{x_{1} ! x_{2} ! \cdots x_{m} !}
$$

a single explicit formula for the coefficient in the first column of our table. $\dagger$ It is also the summation of the coefficients in the symmetric function $(p)$ excepting the factor $(-1)^{m}$, for by (32) and (44), we have

$$
\sum\left(\begin{array}{l}
i \\
p
\end{array}\right)=(-1)^{x_{1}+x_{2}+\cdots+x_{m}} \frac{\left(x_{1}+x_{2}+\cdots+x_{m}\right) !}{x_{1} ! x_{2} ! \cdots x_{m} !},
$$

with $i_{1}+i_{2}+\cdots+i_{m}=m$.

By using the form (40), we can express other coefficients as sums of the form obtained for the first column, when $a_{0}^{m}\left\{i_{m+1} \cdots i_{m+n}\right\}_{t}$ is expanded. If

* We note however the existence of the relation

$$
\frac{|\bar{i}|}{|\lambda|}=\frac{\{\bar{i}\}_{t}}{\{\lambda\}_{t}}=\frac{\{i\}_{a}}{\{\bar{\lambda}\}_{a}}
$$

where the indices of the lines of the determinants are consecutively decreased by unity, and where $\bar{i}$ and $\lambda$ are of order $n$, and $i$ and $\bar{\lambda}$ are of order $m$.

$\dagger$ See footnote to $\S 4(32)$. 
the last quarter of the table has been computed, we have this expansion. * In general, if $\left|{ }_{p}^{i}\right|$ be among the first three-quarters of the coefficients of the table, and $\left|i_{m+1} \cdots i_{2 m}\right|$ heads the conjugate column among the last quarter, then all coefficients in the same column with $\left|{ }_{p}^{i}\right|$ can be found from the function,

and we have,

$$
a_{0}^{m}\left\{i_{m+1} \cdots i_{2 m}\right\}_{t}=\sum\left|\begin{array}{c}
i_{m+1} \cdots i_{2 m} \\
q_{1} \cdots q_{m}
\end{array}\right| t_{q_{1}} t_{q_{2}} \cdots t_{q_{m}}, \dagger
$$

$$
\left|\begin{array}{c}
i_{1} i_{2} \cdots i_{m} \\
p_{1} p_{2} \cdots p_{m}
\end{array}\right|=\sum_{q}(-1)^{m+r_{1}+r_{2}+\cdots}\left|\begin{array}{c}
i_{m+1} \cdots i_{2 m} \\
q_{1} \cdots q_{m}
\end{array}\right| \sum_{r} \frac{r_{1} ! r_{2} ! \cdots}{\rho_{1} ! \rho_{2} ! \cdots \sigma_{1} ! \sigma_{2} ! \cdots},
$$

where $i_{m+1} \cdots i_{2 m}$ are the indices conjugate to $i_{1} i_{2} \cdots i_{m}$, and where $r_{1}, r_{2}, \cdots, \rho_{1}, \rho_{2}, \cdots, \sigma_{1}, \sigma_{2}, \cdots$, are defined as follows : $\epsilon_{1} \epsilon_{2} \cdots \epsilon_{r_{1}} \eta_{1} \eta_{2} \cdots \eta_{r_{2}} \cdots$ is any permutation of $p_{1} p_{2} \cdots p_{m}$ such that

$$
\begin{array}{lll}
\epsilon_{1}+\epsilon_{2}+\cdots+\epsilon_{r_{1}}=q_{1}, & \rho_{1} \text { of the } \epsilon \text { 's are } 1, & \rho_{2} \text { are } 2, \cdots, \\
\eta_{1}+\eta_{2}+\cdots+\eta_{r_{2}}=q_{2}, & \sigma_{1} \text { of the } \eta^{\prime} \text { s are } 1, & \sigma_{2} \text { are } 2, \cdots,
\end{array}
$$

Thus we find for the second column, $(m>1)$,

$$
\left|\begin{array}{c}
02 \cdots m-1 m+1 \\
p_{1} p_{2} \cdots p_{m}
\end{array}\right|=(-1)^{m+1+x_{1}+x_{2}+\cdots+x_{m}} \frac{\left(x_{2}+\cdots+x_{m}\right)\left(x_{1}+\cdots+x_{m}-1\right) !}{x_{1} ! x_{2} ! \cdots x_{m} !}
$$

for the third column, $(m>3)$,

$$
\begin{aligned}
\begin{array}{c}
013 \cdots m-2 m m+1 \\
p_{1} p_{2} \cdots p_{m}
\end{array} \mid & =(-1)^{m+1+x_{1}+\cdots+x_{m}}\left(\frac{\left(x_{1}+\cdots+x_{m}-1\right) !}{\left(x_{1}-1\right) ! x_{2} ! \cdots x_{m} !}\right. \\
& \left.-\frac{\left(x_{1}+\cdots+x_{m}-2\right) !}{\left(x_{1}-2\right) ! x_{2} ! \cdots x_{m} !}-\frac{\left(x_{1}+\cdots+x_{m}-1\right) !}{x_{1} !\left(x_{2}-1\right) ! \cdots x_{m} !}\right),
\end{aligned}
$$

and for the fourth column, $(m>5)$,

$$
\begin{aligned}
& \left|\begin{array}{c}
0124 \cdots m-3 m-1 m m+1 \\
p_{1} p_{2} \cdots p_{m}
\end{array}\right| \\
& (50)=(-1)^{m+1+x_{1}+\cdots+x_{m}}\left(\frac{\left(x_{1}+\cdots+x_{m}-2\right) !}{\left(x_{1}-2\right) ! x_{2} ! \cdots x_{m} !}+\frac{\left(x_{1}+\cdots+x_{m}-1\right) !}{x_{1} !\left(x_{2}-1\right) ! \cdots x_{m} !}\right.
\end{aligned}
$$

* The computation includes the self conjugate column or columns. In the last quarter of the table $\left|\begin{array}{c}i_{m+1} \ldots i_{2 m} \\ q_{1} \ldots q_{m}\end{array}\right|$ is usually $+1,-1$, or 0 , is practically a sign factor in most cases and its value is seen at once by writing down the notation, by means of $\S 3,2$.

†It will be seen that the $t$ 's play here an analogous rôle to that of the s's in the expression of a monomial symmetric function. 


$$
\left.-\frac{\left(x_{1}+\cdots+x_{m}-3\right) !}{\left(x_{1}-3\right) ! x_{2} ! \cdots x_{m} !}-\frac{2\left(x_{1}+\cdots+x_{m}-2\right) !}{\left(x_{1}-1\right) !\left(x_{2}-1\right) ! \cdots x_{m} !}-\frac{\left(x_{1}+\cdots+x_{m}-1\right) !}{x_{1} ! x_{2} !\left(x_{3}-1\right) ! \cdots x_{m} !}\right) \cdot{ }^{*}
$$

\section{$\S 7$. Constructed tables of coefficients.}

1. If $p_{1} p_{2} \cdots p_{n}$ is any partition of weight $w$, the partition $1^{p_{1}-p_{2}} 2^{p_{2}-p_{3}} \cdots n^{p_{n}}$ is the conjugate partition of weight $w$, and it follows that the first is also the conjugate of the second partition. The complex $p_{n}, p_{n-1}+1, \cdots p_{1}+n-1$, and that one got by taking the numbers of the series $012 \cdots m+n-1$, which remain after the complementaries of the former complex with respect to $m+n-1$, have been rejected, are obtained from the preceding conjugate partitions by adding $0,1,2, \cdots n-1$ and $0,1,2, \cdots m-1$ to their respective complexes arranged in ascending order of magnitude, and where $m \geqq p_{1}$. The first complex is evidently obtained as stated. The complementaries of its elements are the factors of the symbolical product of elements

$$
\prod_{1}^{n}\left(m+r-p_{r}-1\right) \text {. }
$$

All other elements but these arranged in ascending order of magnitude are the $n+1$ factors of the product

$$
\prod_{0}^{n}\left(m+r-p_{r} \cdots m+r-p_{r+1}-1\right),
$$

where the $r+1$ th factor is a product of $p_{r}-p_{r+1}$ consecutive elements, and where $p_{0}=m, p_{n+1}=0$ and where any $r+1$ th factor containing negative elements, or elements in descending order is to be rejected. If from the factors of this product we subtract the corresponding factors of $012 \cdots m-1$, similarly expressed as

$$
\prod_{0}^{n}\left(m-p_{r} \cdots m-p_{r+1}-1\right)
$$

a product of $n+1$ factors in which the $(r+1)$ th factor is the product of $p_{r}-p_{r+1}$ consecutive elements, we have for the $(r+1)$ th factor of the remain$\operatorname{der} r^{p_{r}-p_{r+1}}$, and hence the remainder is

$$
\prod_{0}^{n} r^{p_{r}-p_{r+1}}=\prod_{1}^{n} r^{p_{r}-p_{r+1}}=1^{p_{1}-p_{2}} 2^{p_{2}-p_{3}} \ldots n^{p_{n}}, \dagger
$$

as was stated. On this account such complexes are called conjugate complexes, and alternants or determinants which have them for first line indices are called

* In these formulas terms containing negative factorials are to be placed equal to zero.

$\dagger$ The zero elements can be omitted as they merely call attention to the fact that $m-p_{1}$ places in the partition are not filled. 
conjugate alternants or determinants. When $n=m$ they are called principal conjugate alternants or determinants. Every complex has one principal conjugate complex and an infinite number of other conjugate complexes. Analogous statements are true of alternants and determinants. Thus $|1234|$ and $|0127|$ are principal conjugate alternants, $\{1234\}$ and $\{0127\}$ principal conjugate determinants. Among the principal conjugates occur self-conjugate complexes, alternants or determinants. Thus $\{01347\}$ is a self-conjugate determinant.

2. For purposes of tabulation of coefficients, we write in a column at the left the different symmetric functions (partitions) of weight $m$, with conjugate partitions equidistant from the ends, and with the exception of the self-conjugates which will come in the middle, we arrange the upper portion in order from the least to the greatest distribution. That distribution is least, the number of elements being the same, which comes first in lexicographic order (e. g., 52 comes before 43), and the number of elements being different, that which has the least number being first (e. g., 43 comes before $51^{2}$ ). It follows by considering the conjugates of two consecutive partitions, that the lower portion of the column will then be arranged, so that either a partition with the same number of elements as the next comes first in lexicographic order, or one with fewer elements precedes, or if one with more elements comes first, it will contain a greater element than the following ones. For of two consecutive partitions, the complexes are either of the form

$$
\begin{aligned}
& a_{1} a_{2} \cdots a_{\kappa} a_{\kappa+1} \cdots a_{r}, \\
& a_{1} a_{2} \cdots a_{\kappa}-1 a_{\kappa+1}+1 \cdots a_{r},
\end{aligned}
$$

and the respective conjugates will occur in the order,

$$
\begin{aligned}
& 1^{a_{1}-a_{2}} 2^{a_{2}-a_{3}} \cdots(\kappa-1)^{a_{\kappa-1}+1-a_{\kappa}} \kappa^{a_{\kappa}-2-a_{\kappa+1}}(\kappa+1)^{a_{\kappa+1}+1-a_{\kappa+2}} \ldots r^{a_{r}}, \\
& 1^{a_{1}-a_{2}} 2^{a_{2}-a_{3}} \cdots(\kappa-1)^{a_{\kappa-1}-a_{\kappa}} \kappa^{a_{\kappa}-a_{\kappa+1}} \cdots r^{a_{r}},
\end{aligned}
$$

or they are of the form

$$
\begin{aligned}
& a_{1} a_{2} \cdots a_{r}, \\
& a_{1}+1 a_{2} \cdots a_{\kappa}-1 \cdots a_{i}-1 \cdots a_{r+1},
\end{aligned}
$$

and their conjugates will occur as

$$
\begin{aligned}
& 1^{a_{1}+1-a_{2}} 2^{a_{2}-a_{3}} \cdots \kappa^{a_{\kappa}-1-a_{\kappa+1}} \ldots i^{a_{i}-1-a_{i+1}} \cdots(r+1)^{a_{r+1}}, \\
& 1^{a_{1}-a_{2}} 2^{a_{2}-a_{3}} \cdots \kappa^{a_{\kappa}-a_{\kappa+1}} \cdots i^{a_{i}-a_{i+1}} \cdots r^{a_{r}} .
\end{aligned}
$$

In the first case a conjugate partition with the same number $\left(a_{1}\right)$ of elements lexicographically or with fewer elements (one less when $\kappa=1$ ), in the second case one having a greater element $(r+1)$ precedes. 
We next write in a horizontal line the same partitions, beginning at the bottom of the column and setting them down in the reverse column order from right to left. Above each one we place the complex obtained by adding $012 \cdots m-1$ to its elements, zeros supplied to bring the number up to $m$, and all arranged in ascending order of magnitude before adding. These complexes give the indices of all the alternants possible in all of the products $A \phi$, where $\phi$ is any monomial symmetric function of weight $m$, and $A=|012 \cdots m-1|$, or also the first line indices of all determinants possible in all products $a_{0}^{m} \phi$, with conjugate complexes equidistant from the ends. Since the different partitions are the diagonal indices of $\S 3,2$, the coefficient of any alternant which corresponds to a partition earlier than the function is zero, because the lower complex will contain either fewer or greater elements, hence by the operation of $\S 3,2$ it is impossible to make the lower equal to the upper complex; for, in the one case, while that operation can diminish, it can not increase the number of elements below, and in the other, while it can increase the element in the last place by passing it over preceding elements, it can not diminish it. Thus we have generally proved, if $q_{1} q_{2} \cdots q_{m}$ precede $p_{1} p_{2} \cdots p_{m}$,

$$
\left[\begin{array}{l}
p_{1} p_{2} \cdots p_{m} \\
q_{1} q_{2} \cdots q_{m}
\end{array}\right]=0
$$

The last coefficient which can have significance in a horizontal line is evidently the one

$$
\left[\begin{array}{l}
p_{1} p_{2} \cdots p_{m} \\
p_{1} p_{2} \cdots p_{m}
\end{array}\right]=1
$$

The formulas (56) and (57) taken together show that our coefficients all lie in a triangle of the table, with the bounding diagonal of ones for its hypothenuse.*

3. The sum of the coefficients in every column is zero, except for the last column which contains only unity. Since $\left|\begin{array}{c}\kappa \\ p\end{array}\right|=\left\{\begin{array}{c}p \\ \kappa\end{array}\right\}$, the coefficients of the column headed by the complex $\kappa$, are the different coefficients in the development of $\{\kappa\}$. If the $a$ 's are put equal to unity $\{\kappa\}=0$, except for the last column, where $\{012 \cdots m-22 m-1\}=1$; hence we have proved,

$$
\sum_{p}\left|\begin{array}{l}
\kappa_{1} \kappa_{2} \cdots \kappa_{m} \\
p_{1} p_{2} \cdots p_{m}
\end{array}\right|=0
$$

where the $\kappa$ 's are any complex of column indices, but the last, and the $p$ 's run through all partitions of weight $m$. This property affords a useful check in computing the tables. In accordance with the construction here explained and

* The triangle property would still hold for a table arranged in strict dictionary order, for then by $\$ 3,2\left[\begin{array}{l}p \\ q\end{array}\right]=0$, if $q$ precedes $p$. 
by the various methods of computing previously discussed, tables are given in this paper up to weight seven.

\section{§ 8. On the quotient of two alternants.}

The coefficients of any symmetric function in the quotient of an alternant $|\kappa|$ by the alternant $|012 \cdots m-1|$, both being of order $m$, can be found as follows :

1. If the given alternant is in the table, let $r$ be the number of removes of the symmetric function from the last line in which the alternant has a coefficient. Let $s$ be the number of partitions in the table from this line to the end. Then the coefficient of the symmetric function in the quotient

$$
\frac{\left|\kappa_{1} \kappa_{2} \cdots \kappa_{m}\right|}{|012 \cdots m-1|} \quad \text { is } \quad(-1)^{s-r+\frac{s(s-1)}{2}}
$$

times the determinant formed by taking the next $r-1$ lines $(r-i+1$ elements at the end from the $i$ th of these lines) and for the last line $r$ elements immediately above the $r$ elements first chosen, with their signs changed. Thus the coefficient of $\left(31^{3}\right)$ in the quotient,

$$
\left.\frac{|012369|}{|012345|} \text { is }(-1)^{8-4+28}\left|\begin{array}{rrrr}
1 & -1 & 0 & 1 \\
-1 & -1 & 1 & 0 \\
-2 & 1 & 0 & 0 \\
-1 & 0 & 1 & 1
\end{array}\right|=3 \text { (see } \S 4\right) \text {. }
$$

In fact if we multiply the different symmetric functions $\phi_{1}, \phi_{2}, \ldots$ involved by undetermined multipliers $\mu_{1}, \mu_{2}, \ldots$ and then so determine the $\mu$ 's that

$$
|012 \cdots m-1| \sum \mu \phi=|\kappa|
$$

we shall obtain the result stated.

2. If the given alternant is not in a table, we take the difference

$$
\kappa_{1} \kappa_{2}-1 \kappa_{3}-2 \cdots \kappa_{m}-m+1
$$

of the complexes in the dividend and divisor and note the weight and partition of the complex, then we turn to the table of the given resulting weight, and take the column headed by the resulting partition and proceed exactly as in case 1. The explanation of this will appear in the next section. Thus the coefficient of $\left(31^{3}\right)$ in $|012 \cdots m m+3| /|012 \cdots m-1|$ is 3 .

\section{§ 9. On the invariance of the coefficients $\left|\begin{array}{l}i \\ p\end{array}\right|$.}

In $\S 7$ we have supposed the alternant $|012 \cdots m-1|$ to be the multiplier of the symmetric functions of weight $m$. We will now show that the whole 
product table for weight $m$ remains absolutely invariant and still gives the complete product, if the column headings be merely altered suitably, when $|012 \cdots m+r-1|$ is used as multiplier. In fact by $\S 3,2$,

$$
\left|\begin{array}{c}
\kappa_{1} \kappa_{2} \cdots \kappa_{m+r} \\
0^{r} p_{1} p_{2} \cdots p_{m}
\end{array}\right|=\left[\begin{array}{c}
0^{r} p_{1} p_{2} \cdots p_{m} \\
\kappa_{1} \kappa_{2}-1 \cdots \kappa_{m+r}-m-r+1
\end{array}\right] \text {. }
$$

To be significant the lower complex on the right hand side must be of weight $m$, must therefore contain at least $r$ zeros $\kappa_{1} \kappa_{2}-1 \ldots \kappa_{r}-r+1$, and is some partition of weight $m$. Of such partitions however only those of the table exist, hence omitting zeros,

$$
\left|\begin{array}{l}
\kappa_{1} \kappa_{2} \cdots \kappa_{m+r} \\
0^{r} p_{1} p_{2} \cdots p_{n n}
\end{array}\right|=\left[\begin{array}{l}
p_{1} p_{2} \cdots p_{m} \\
\kappa_{r+1}-r \cdots \kappa_{m+r}-m-r+1
\end{array}\right],
$$

where the right hand member of $(60)$ is a coefficient of the table. By adding the elements of a partition of the horizontal line arranged in ascending order to the same number of elements at the end of $012 \cdots m+r-1$, we obtain the new column heading. Thus the table for weight $w$ is a table for an infinite number of products.*

If the multiplier is $|012 \cdots m-r-1|$, then it is evident as before, that the table still gives the coefficients for those columns headed by a partition not containing more than $m-r$ elements, but that for the rest the multiplication will have to be indicated. From the invariance of the product table follows also the invariance of the quotient table.

Examples :

1. To find $|0123456789|\left(321^{2}\right)$.

We turn to the table of weight seven, and add $1^{7}, 21^{5}, 2^{2} 1^{3}, 2^{3} 1,31^{4}, 321^{2}$, to 0123456789 and obtain the complexes of the alternants in the product, and in the line $321^{2}$, the respective coefficients and have,

$|0123456789|\left(321^{2}\right)=-12|01245678910|+6|01235678911|$

$$
+|012346781011|-2|012345791011|-3|01234678912|
$$

$$
\begin{aligned}
& +|012345781012| . \\
& |01|(4)=-|14|+|05|+\sum_{3}^{m} \alpha_{\kappa}|01| .
\end{aligned}
$$

* This invariance also appears from the standpoint that the table is also a table for symmetric functions. Using different alternants as multipliers in the manner indicated is equivalent, by the theorem of corresponding matrices, to multiplying the function by different powers of $a_{0}$, which can not affect the coefficients, since, as is well known, they are not functions of the powers of $a_{0}$.

The tahle also retains its invariance when the multiplier is $|\lambda, \lambda+1, \cdots m+r+\lambda-1|$, i. e., when each of the indices of $|012 \cdots m+r-1|$ is increased by the same positive integer $\lambda$. 
If we limit example 2 to a quadratic, $\alpha_{3}=\alpha_{4}=\cdots=\alpha_{m}=0$, and the product is complete with the first two terms; this is another way of looking at this case.

\section{§10. The table as a table for symmetric functions.}

By (6), the second of the theorems mentioned in $\S 1$, our table becomes a table for the expression of the symmetric function $a_{0}^{m}(p)$ as a sum of determinants of the $a$ 's of the $m$ th order when conjugate column complexes are interchanged, and all the coefficients have been multiplied by $(-1)^{10} .^{*}$ As the coefficients in the expansion of the given determinants are already found in the columns headed by their first line complexes of indices, we have only to take these coefficients with their partitions at the left interpreted as $a$ 's, and multiply by the coefficient of the determinant and take the sum of all such determinants in order to get $a_{0}^{m}(p)$. Thus our table becomes a table for expressing $(p)$ in terms of elementary products (cf. $\S 1$ ).

Examples :

1. To find $a_{0}^{2}\left(2^{2} 1^{2}\right)$. From the table of weight six we find

$$
a_{0}^{6}\left(2^{2} 1^{2}\right)=6\{0123411\}-3\{0123510\}+\{012369\} .
$$

By the same table:

$$
\begin{aligned}
\{0123411\} & =a_{0}^{5} a_{6}, \\
\{0123510\} & =-a_{0}^{5} a_{6}+a_{0}^{4} a_{1} a_{5}, \\
\{012369\} & =-a_{0}^{4} a_{1} a_{5}+a_{0}^{4} a_{2} a_{4} .
\end{aligned}
$$

Introducing these values in (63) and dividing by $a_{0}^{4}$, we get

$$
a_{0}^{2} \sum \alpha_{1}^{2} \alpha_{2}^{2} \alpha_{3} \alpha_{4}=9 a_{0} a_{6}-4 a_{1} a_{5}+a_{2} a_{4} .
$$

2. To find $\left(31^{2}\right)$. Put $a_{0}=1$; from the table of weight 5 , by changing the signs of all the coefficients, since $(-1)^{x}=(-1)^{5}=-1$, we have proceeding as before,

$$
\left(31^{2}\right)=-3\{01239\}+\{01248\}+\{01257\}-\{01347\}
$$

$$
\sum \alpha_{1}^{3} \alpha_{2} \alpha_{3}=-5 a_{5}+a_{1} a_{4}+2 a_{2} a_{3}-a_{1}^{2} a_{3} .
$$

\footnotetext{
* If the column complexes and the coefficients are used unchanged, then $(p)$ is expressed as a sum of determinants in the $t^{\prime} \mathrm{s}$, on account of the relations (41), \& 5, and the expansion of these determinants is given by the table by interpreting the partitions at the left as $t$ 's.
} 
§11. Seven product tables.

We give here product tables from weight one to weight seven inclusive.
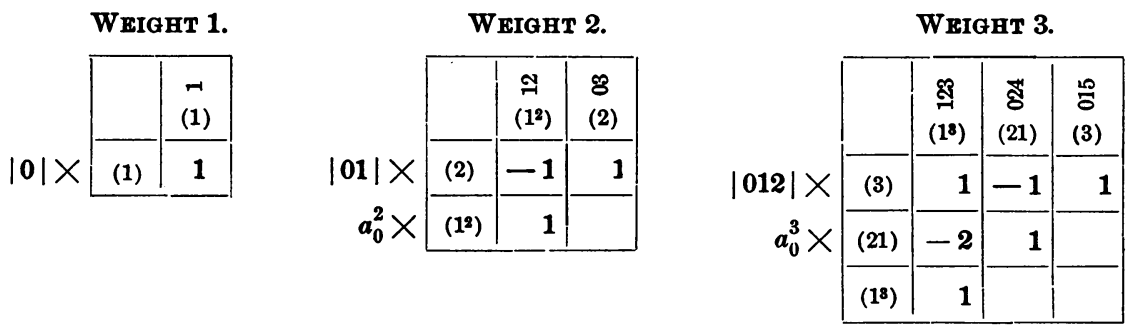

WEIGHT 4.

\begin{tabular}{|c|c|c|c|c|c|c|}
\hline \multirow{6}{*}{$\begin{array}{r}|0123| \times \\
a_{0}^{4} \times\end{array}$} & & 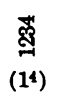 & $\begin{array}{c}\text { कू } \\
(212)\end{array}$ & $\begin{array}{l}\stackrel{8}{0} \\
\left(2^{2}\right)\end{array}$ & $\begin{array}{l}\stackrel{0}{\circ} \\
\text { g. } \\
(31)\end{array}$ & $\begin{array}{l}\text { 청 } \\
\text { (4) }\end{array}$ \\
\hline & (4) & -1 & $\mathbf{1}^{\prime}$ & 0 & -1 & 1 \\
\hline & (31) & 2 & $-\mathbf{i}$ & -1 & 1 & \\
\hline & $\left(2^{2}\right)$ & 1 & -1 & 1 & & \\
\hline & $\left(21^{2}\right)$ & -3 & 1 & & & \\
\hline & $\left(1^{4}\right)$ & 1 & & & & \\
\hline
\end{tabular}

WEIGHT 5.

\begin{tabular}{|c|c|c|c|c|c|c|c|c|}
\hline \multirow{8}{*}{$\begin{array}{r}|01234| \times \\
a_{0}^{5} \times\end{array}$} & & 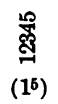 & $\begin{array}{c}\text { \%ัँ } \\
\text { क्ष } \\
\left(21^{8}\right)\end{array}$ & $\begin{array}{c}\text { 呬 } \\
\text { (21) } \\
(21)\end{array}$ & 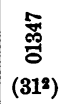 & 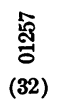 & $\begin{array}{c}\text { 유 } \\
\text { त्र } \\
(41)\end{array}$ & 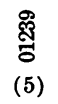 \\
\hline & (5) & 1 & -1 & 0 & 1 & 0 & -1 & 1 \\
\hline & (41) & -2 & 1 & 1 & -1 & -1 & 1 & \\
\hline & (32) & --2 & 2 & -1 & -1 & 1 & & \\
\hline & $\left(31^{2}\right)$ & 3 & -1 & -1 & 1 & & & \\
\hline & $\left(2^{21}\right)$ & 3 & -2 & 1 & & & & \\
\hline & $\left(21^{8}\right)$ & -4 & 1 & & & & & \\
\hline & $\left(1^{6}\right)$ & 1 & & & & & & \\
\hline
\end{tabular}


WEIGHT 6.

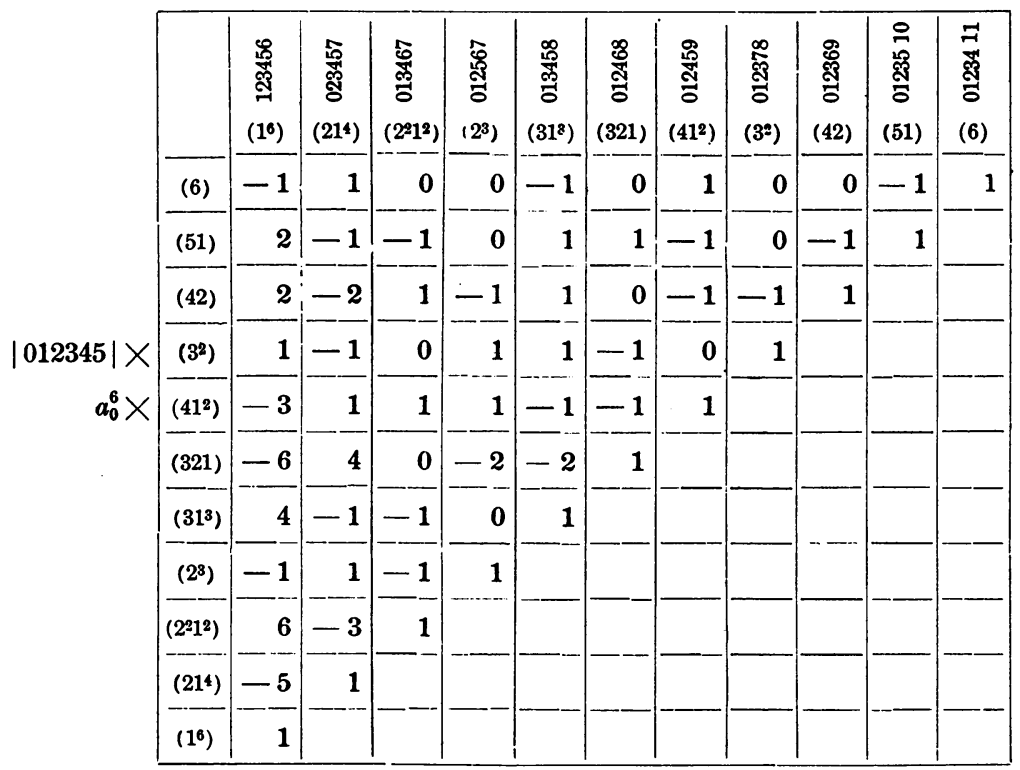

WEIGHT 7.

\begin{tabular}{|c|c|c|c|c|c|c|c|c|c|c|c|c|c|c|c|}
\hline & 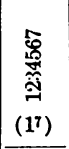 & 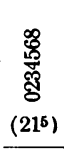 & 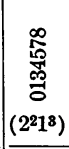 & 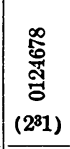 & 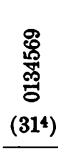 & 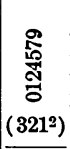 & 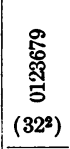 & 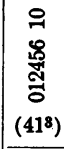 & 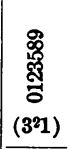 & 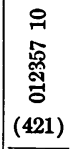 & 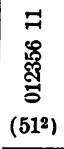 & 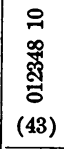 & $\begin{array}{c}7 \\
7 \\
\text { స్ } \\
\text { ్ㅐ } \\
(52) \\
\end{array}$ & 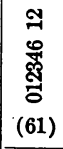 & 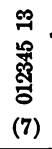 \\
\hline (7) & 1 & -1 & 0 & 0 & 1 & 0 & 0 & -1 & 0 & 0 & 1 & 0 & 0 & -1 & 1 \\
\hline (61) & -2 & 1 & 1 & 0 & -1 & -1 & 0 & 1 & 0 & 1 & -1 & 0 & -1 & 1 & \\
\hline (52) & -2 & 2 & -1 & 1 & -1 & 0 & -1 & 1 & 1 & 0 & -1 & -1 & 1 & & \\
\hline (43) & -2 & 2 & 0 & -1 & -2 & 1 & 1 & 1 & -1 & -1 & 0 & 1 & & & \\
\hline$\left(51^{2}\right)$ & 3 & -1 & -1 & -1 & 1 & 1 & 1 & -1 & 0 & -1 & 1 & & & & \\
\hline (421) & 6 & -4 & 0 & 0 & 2 & 1 & -1 & -2 & -1 & 1 & & & & & \\
\hline$\left(3^{21}\right)$ & 3 & -2 & $\mid-1$ & 2 & 2 & -1 & -1 & 0 & 1 & & & & & & \\
\hline$\left(41^{8}\right)$ & -4 & 1 & 1 & 1 & -1 & -1 & 0 & 1 & & & & & & & \\
\hline$\left(32^{2}\right)$ & 3 & -3 & 2 & -1 & 1 & -1 & 1 & & & & & & & & \\
\hline$\left(321^{2}\right)$ & -12 & 6 & 1 & -2 & -3 & 1 & & & & & & & & & \\
\hline$\left(31^{4}\right)$ & 5 & -1 & -1 & 0 & 1 & & & & & & & & & & \\
\hline$\left(2^{31}\right)$ & -4 & 3 & -2 & 1 & & & & & & & & & & & \\
\hline$\left(2^{2} 1^{3}\right)$ & 10 & -4 & 1 & & & & & & & & & & & & \\
\hline$\left(21^{5}\right)$ & -6 & 1 & & & & & & & & & & & & & \\
\hline$\left(1^{7}\right)$ & 1 & & & & & & & & & & & & & & \\
\hline
\end{tabular}

